\title{
Facial Expression Recognition Using Static Facial Images
}

\author{
G.Sowmiya $^{*}$, V. Kumutha ${ }^{2}$ \\ ${ }^{1}$ Department of Computer Science, Sri Krishna Arts and Science College, Coimbatore, India \\ ${ }^{2}$ Department of Computer Science, Sri Krishna Arts and Science College, Coimbatore, India \\ *Corresponding Author: sowmiyag16mcs06@skasc.ac.in, Tel.: +91-9585960821
}

Available online at: www.isroset.org

Received: 27/Feb/2018, Revised: 12/Mar/2018, Accepted: 31/Mar/2018, Online: 30/Apr/ 2018

\begin{abstract}
Delicate concerns about the treatment of individuals during interviews and interrogations have stimulated efforts to develop "non-intrusive" technologies for rapidly assessing the credibility of statements by individuals in a variety of sensitive environments. Methods or processes that have the potential to exactly focus investigative resources will advance operational excellence and improve investigative capabilities. Facial expressions have the capacity to communicate emotion and regulate interpersonal behavior. Facial Expression Recognition -FER has been dramatically developed in recent years, especially machine learning, Image processing and human cognition. For this reason, the bang and possible usage of automatic facial expression recognition system have been mounting in a broad range of applications, including human-computer interaction, robot control and driver state observation. This paper proposes an automatic facial expression recognition using static facial images, capable of distinctive the four universal emotions: neutral, happiness, sadness and surprise. It is designed to be person independent and tailored only for static images.
\end{abstract}

Keywords Facial Expression recognition, eye and lip detection, Bezier curve, emotion, RGB color, binary image pixel.

\section{INTRODUCTION}

In the developing technology emotion recognition from the facial expression is one of the most important research areas. During the analysis process, one of the major challenges there in the system is wide range of variation in the human face image which leads to reduce the entire system performance while analyzing the face related emotions. So, the automatic facial related emotion recognition using static images is implemented using the different image processing and machine learning techniques. Initially the face image has been capture and the image is analyzed according to the skin color. After the color image is transmitted to the grey scale image and the noise present image is eliminated with the help of the non-local median filtering approach.

Facial expression is a visible manifestation of the affective state, cognitive activity, intention, personality, and Psychopathology of a person [5]; it plays a communicative role in interpersonal relations. Facial expressions, and other gestures, transmit non-verbal communication cues in face-toface interactions. These cues may also complement speech by helping the listener to elicit the intended meaning of spoken words. The facial expression of a speaker accounts for about
55 percent of the effect, 38 percent of the latter is conveyed by voice intonation and 7 percent by the spoken words. The main goal of this paper is researching existing methods for performing automatic facial expression recognition. This is enforced by developing a system which is capable of classifying an image into one of the four basic emotions.

Facial expression recognition (FER) is currently a very active research topic in the fields of computer vision, pattern recognition, artificial intelligence, and has drawn extensive attentions owing to its potential applications to natural human-computer interaction (HCI) [6], human emotion analysis, interactive video, image indexing and retrieval, etc. In computer vision, a facial expression is usually considered as the deformations of facial components and their spatial relations, or changes in the pigmentation of the face. Facial expression recognition using static facial images is a computer system that attempts to categorize these changes or deformations into abstract classes robotically [4].

\section{PROCESS OF FACIAL EXPRESSION RECOGNITION}


In this section discusses about the facial expression related emotion identification process which includes the several stages such as skin color segmentation that perform the contrasting then segment the skin color, face detection that detect the face which will contain only eyes, nose and lip, eye detection that detect the eyes for scan from the W/4 to (W-W/4) to find the middle position of the two eyes, lip detection that will contain only lip and may some part of the nose. Then we will cut the RGB image according the lip shape, apply the Bezier curve on the eye and lip. Then we get the shape of the eye and lip and emotion detection that convert each width (eye and lip) of the Bezier curve to 100 and height according to its width.

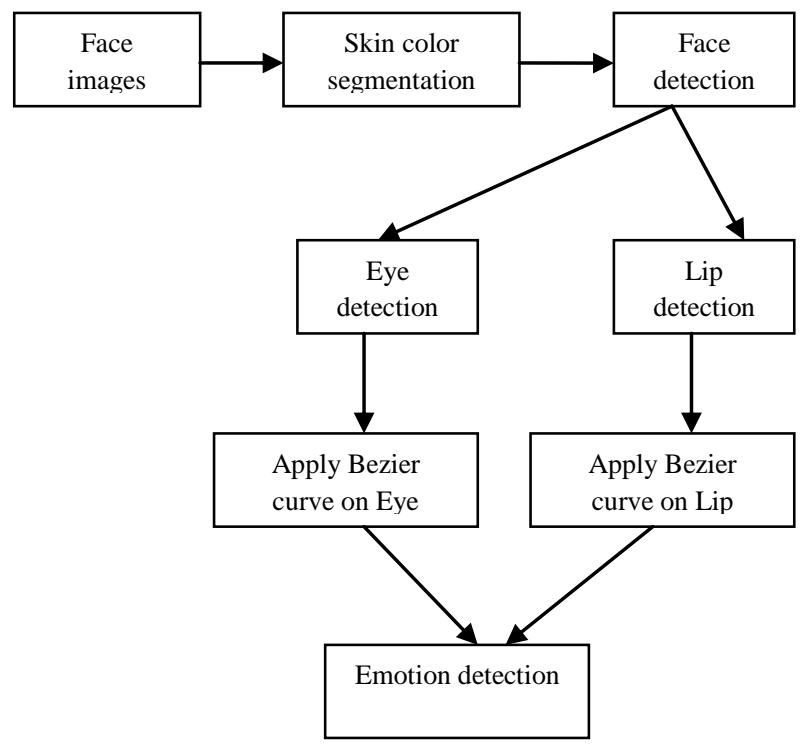

Figure 1: Proposed Expression Recognition System Architecture

\section{SKIN COLOR SEGMENTATION}

Segmentation is the categorization of the input colored image into skin and non-skin pixels based on skin color information. A wide range of applications that require the segmentation process as a preprocessing operation such as computer vision, face/ hand detection and recognition, medical image analysis, and pattern recognition. Color information is one of the simple cues used for detecting skin color, and the use of proper color space to represent color information of an image is a crucial decision [1].

Before the segmentation of skin color, we want to contrast the image. Contrast can be simply explained as the difference between maximum and minimum pixel intensity in an image. Contrast is the visual difference that makes an object distinguishable from background and other objects [11]. For better and easier human interpretation of images and higher perceptual quality, contrast enhancement becomes necessary.

In this paper, RGB color model is used for skin color segmentation. RGB color space is broadly used and is normally the default color space for storing and representing digital images [12]. We can get any other color space from a linear or non-linear conversion of RGB. The RGB color model is the color component used by computers, graphics cards and monitors or LCDs. Depending on how much is taken from each base color, any color can be created. Reversing this technique, a particular color can be broken down into its red, blue and green components as shown in equation 1 to equation 3 . These values can be used to find out similar colored pixels from the image.

$$
\begin{aligned}
& r=\frac{R}{R+G+B} \\
& g=\frac{G}{R+G+B} \\
& b=\frac{B}{R+G+B}
\end{aligned}
$$

\section{FACE DETECTION}

Face detection has been the most important phase in identifying facial expressions. To detect a face has been more difficult and since then more methods have been evolving to simplify the task. One such method is to first segment the image based on the skin area and then to detect the face. Face detection is the primary step in building a practical FER system. Skin color segmentation is used to detect face(s) from the static images.

Connected component operators are non-linear filters that eliminate parts of the image, while preserving the contours of the remaining parts. This simplification property makes them attractive for segmentation and pattern recognition applications [1]. 
After connect the region, the RGB image converted into binary image for perfect face detection that contains eyes, lip and nose only. It is significant in image processing to extort objects from their environment into binary image. Binary image is used for feature extraction process and have an essential role in generating exclusive feature [8]. In the image processing, image binarization is used as a common tool for image segmentation of selective objects from backdrop in a variety of applications such as automatic target tracking, object detection, image compression, image analysis, and object partition
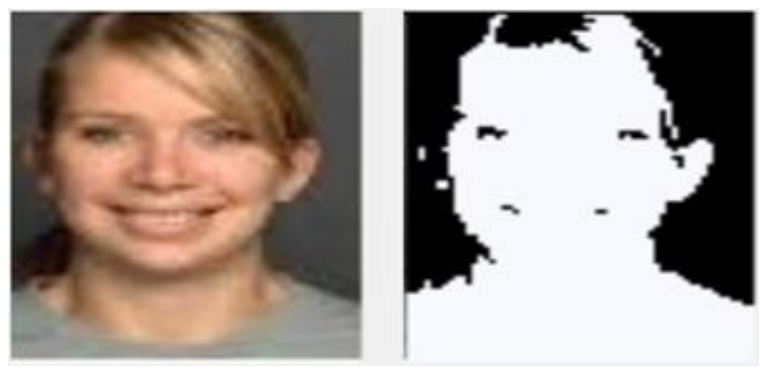

Figure 2: The original image and generated binary image

\section{EYE AND LIP DETECTION}

Recognition of facial appearance for example eye, nose, and mouth is an essential step for many following facial image analysis tasks for example face recognition. In this paper, we commence a technique to detect eye and nose fields from gray scale facial images. Eye detection is critical whenever we are dealing with face analysis and recognition of facial features. Eye detection is somewhat difficult due to multiple variations in human faces, size of eyes and due to eyeglasses.

\section{Eye detection:}

In this paper, for eyes detection, we convert the RGB face to the binary face. Now, we consider the face width by $\mathrm{W}$. We scan from the $\mathrm{W} / 4$ to $(\mathrm{W}-\mathrm{W} / 4)$ to find the middle position of the two eyes. The maximum white continuous pixel alongside the height between the ranges is the center location of the two eyes. Then find the initial high or upper position of the two eyebrows by searching vertically. For left eye, search w/8 to mid and for right eye, search mid to $w-$ $\mathrm{w} / 8$. Here $\mathrm{w}$ is the width of the image and mid is the center position of the two eyes. There may be some white pixels between the eyebrow and the eye. To compose the eyebrow and eye connected, put some continuous black pixels vertically from eyebrow to the eye.
For left eye, the vertical black pixel-lines are located in between $\mathrm{mid} / 2$ to $\mathrm{mid} / 4$ and for right eye the lines are in the center of mid+(w-mid)/ 4 to $\mathrm{mid}+3 *(\mathrm{w}-\mathrm{mid}) / 4$ and height of the black pixel-lines are from the eyebrow starting height to (h- eyebrow starting position)/4. Here $w$ is the width of the image and mid is the middle position of the two eyes and $\mathrm{h}$ is the height of the image. Next to get the lower location of the two eyes, want to search black pixel vertically. For left eye, looks from the $\mathrm{mid} / 4$ to mid - mid/4 width.

For right eye, explore mid $+(\mathrm{w}-\mathrm{mid}) / 4$ to $\mathrm{mid}+3 *(\mathrm{w}-$ mid)/ 4 width from image lower end to initial position of the eyebrow. Then find the right part of the left eye by searching black pixel horizontally from the mid position to the starting position of black pixels in between the upper position and lower position of the left eye. Left part for right eye search mid to the initial position of black pixels in between the upper location and lower location of right eye. The left side of the left eye is the opening width of the image and the right side of the right eye is the ending width of the image. Final step of the eye detection is cut the upper position, lower position, left side and the right side of the two eyes from the RGB image.

\section{Lip detection:}

In this paper, for lip detection, first establish the distance between the forehead and eyes. Then add the distance with the lower height of the eye to determine the upper height of the lip. Now, the starting point of the lip will be the $1 / 4$ position of the left eye and ending point will be the $3 / 4$ position of the right eye. And the ending height of the lip will be the lower end of the face image. So, this will contain only lip and may some part of the nose. Then we will cut the RGB image according the calculation.

So, for recognition of eyes and lip, only need to transfer binary image from RGB image and some searching between the binary images.

\section{BEZIER CURVE ON EYE AND LIP}

This paper introducing procedure to detect the human expression by using Bezier curve. First, it takes skin color segmented image, it produces human skin color, and then it senses human face. The image is transformed into binary image, Then it detach the eyes \& lip from the face. Then it drawn Bezier curve for eyes \& lips. Then it evaluates the Bezier curve of eyes and lips and stores the curve parameters to the database that are used to find emotion. 
Bezier curves are making use of drawn smooth curves beside points on a path. In this paper, we are using cubic Bezier curve. The cubic Bezier curve is adequate for most graphics application. The cubic Bezier curve has four control points for smoothly extended curve [7], we cannot add additional point to the Bezier curve but we can construct another curve by picking four points and then attach that curve to first [13]. The curves begins at the first control point and end at fourth if we want to connect two Bezier curve we just make the first control point of the second curve, match the last control point of the first curve. at the start of the curve it is tangent to the line connecting the first and the second control point. Similarly at the end of the curve it is tangent to the line connecting third and fourth control point.

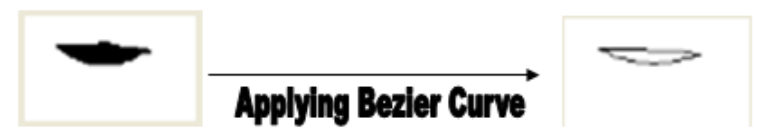

Figure 3: apply Bezier curve on binary lip image

From the fig above the Bezier curve for lip will get the shape of lip. After applying Bezier curve will detect the emotion.

\section{CONCLUSION}

The proposed solution delivers a recognizer system for facial expressions. This paper proposes the one basic technique for facial expression recognition using static facial emotions. In this paper, skin color segmentation, Bezier curve and binary parameters were successfully extracted for four different expressions (neutral, happiness, sadness and surprise). In this paper, the face will be detected from the static images by skin color segmentation process. And finally emotion will be detected by calculating the lip and eyes parameters using Bezier curve technique. The future scope of this system is to detect the more expressions from the static images and detect the expressions from the dynamic images which can be used for medical to detect mental disorders and robotic fields.

\section{REFERENCES}

[1] Noor A. Ibraheem, Rafiqul Z. Khan, Mokhtar M. Hasan. Comparative Study of Skin Color based Segmentation Techniques. August 2013 -IJAIS.

[2] Saket S Kulkarni, Narender P Reddy, and SI Hariharan. Facial expression (mood) recognition from facial images using committee neural networks. August 2009-Biomed central.

[3] Andreea Pascu, Prof. Ross King. Facial expression recognition system, April 2015.
[4] Ce Zhan, Wanqing Li, Philip Ogunbona, and Farzad Safaei. A RealTime Facial Expression Recognition System for Online Games. Hindawi-2008.

[5] Claude C. Chibelushi, Fabrice Bourel. Facial Expression Recognition: A Brief Tutorial Overview.

[6] V.Sathya, T.chakravarthy. Facial Expression Recognition System Performance Using Various Database. Sep.- Oct. 2017- IOSRJCE.

[7] C.Karuna Sharma, T.Aswini, A.Vinodhini, V.Selvi. Accurate Emotion Detection of Digital Images Using Bezier Curves. IJARCET-3, March 2015.

[8] Ratri Dwi Atmaja, Muhammad Ary Murti, Junartho Halomoan, Fiky Yosef Suratman. An Image Processing Method to Convert RGB Image into Binary. IJEECS, August 2016, pp. 377 - 382.

[9] Manish Dixit and Sanjay Silakari. Face Recognition Using Approximated Bezier Curve and Supervised Learning Approach. IJMUE-2015, pp.311-24.

[10] P. Rai and M. Dixit, "Smile detection Via Bezier Curve Of Mouth Interest Points", International Journal of Advanced Research in Computer Science and Software Engineering, vol. 3, no. 7, (2013) July, pp. 802-806.

[11] Ms. K.T.Chalekar, Prof T. Yengantiwar. REVIEW PAPER ON IMAGE CONTRAST ENHANCEMENT TECHNIQUES. (IJARCET) Volume 3 Issue 3, March 2014.

[12] S. Kolkur, D. Kalbande, P. Shimpi, C. Bapat, and J. Jatakia. Human Skin Detection Using RGB, HSV and YCbCr Color Models.

[13] Prof. Rahul, Mangesh, Viplove Wahane, Akshay Murkhe. Analysis of Emotion Recognition using Facial Expressions, using Bezier curve. IJLTET. 